\title{
Large Eddy Simulation of Confined Turbulent Round Jet with Annular Jets
}

\author{
Christian Lagarza, Martín Salinas, Eliseo Martínez, Jorge Ramírez \\ Ciudad Universitaria, Engineering Institute/UNAM
}

Av. Universidad 3000, Circuito Exterior S/N Delegación Coyoacán, C.P. 04510, México City, México

CLagarzaC@iingen.unam.mx; MSalinasV@iingen.unam.mx; EMartínezE@iingen.uanm.mx

\begin{abstract}
Particular geometry is used to simulate numerically a confined turbulent jet. The purpose of this work is evaluate numerically the effect of annular jets above a central jet and define features of turbulence by analysis of anisotropy turbulence. Threedimensional large eddy simulation was used for turbulence modelling. Lumley-Newman triangle representation of anisotropy turbulence was used to define differences between numerical measures of local turbulence and 3D isotropic turbulence state.
\end{abstract}

Keywords: LES, round jet, annular jet, confined, turbulence, anisotropy.

\section{Introduction}

In many industrial applications, mixtures are produced by jets. Generally this kind of flow has two features, the turbulent flow and it is confined. Considering the effect of the surrounding enclosure or confinement a jet presents significant behavior changes. Unlike the free jet sufficient amount of surrounding fluid is not present for the entrainment in confined jet [1]. This develop a recirculation flow pattern. A free jet and confined jet, are quite different (Figure1). In addition the pipe diameter ratio $\left(D_{d} / D_{j}\right)$ present another important feature. Diameter ratios lower than 11 refers to a strong confinement [2].
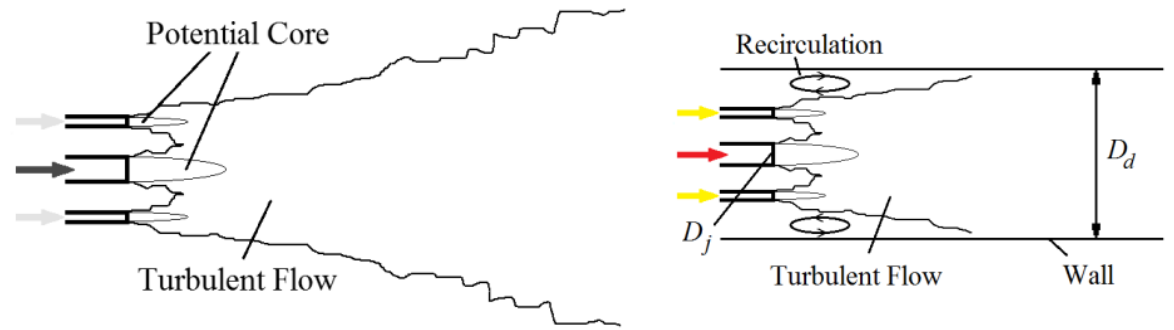

Fig. 1: Difference between an array of free turbulent jets and confined turbulent jets.

Although there are studies about the turbulence of confined jets $[3,4,5,6,7]$, information related with strong confined turbulent jet when it is affected by annular jets is null. Therefore, the purpose of this work is to analyse the effect of confinement and peripheral jets about the turbulence of main jet. This becomes relevant when process tend to be performed in confined or restricted spatial regions.

\section{Governing equations}

In a frame of Cartesian reference compressible Navier-Stokes equations can be written in the form

$$
\frac{\partial U}{\partial t}+\frac{\partial F_{i}}{\partial x_{i}}=S
$$

$U$ is a vector of five component defined by $U=\left(\rho, \rho u_{1}, \rho u_{2}, \rho u_{3}, \rho e\right)^{T}$. It also considers that $u=\left(u_{1}, u_{2}, u_{3}\right)$ is the velocity vector and $\rho$ is the density. Equation 1 shows the evolution of the density, momentum and total energy. $e$ is 
defined as an ideal gas by two terms. First, $C_{v} T$ of internal energy and second, $\frac{1}{2}\left(u_{1}^{2}+u_{2}^{2}+u_{3}^{2}\right)$ of kinetic energy. $F_{i}$ are fluxes where $\forall i \in\{1,2,3\}$ and for a Newtonian fluid are given by

$$
F_{i}=\left[\begin{array}{c}
\rho u_{i} \\
\rho u_{i} u_{1}+\rho \delta_{i 1}-2 \mu S_{i 1} \\
\rho u_{i} u_{2}+\rho \delta_{i 2}-2 \mu S_{i 2} \\
\rho u_{i} u_{3}+\rho \delta_{i 3}-2 \mu S_{i 3} \\
(\rho e+p) u_{i}-2 \mu u_{j} S_{i j}-k \frac{\partial T}{\partial x_{i}}
\end{array}\right]
$$

$k=\rho C_{p} K$ is the thermal conductivity and $K$ is the thermal diffusivity. The symbol $\delta_{i j}$ is the Kronecker delta and $S_{i j}$ is the deviatoric component of strain tensor. The term $S_{i j}$ is written as

$$
S_{i j}=\frac{1}{2}\left(\frac{\partial u_{i}}{\partial x_{j}}+\frac{\partial u_{j}}{\partial x_{i}}-\frac{2}{3}(\nabla \cdot u) \delta_{i j}\right)
$$

Molecular viscosity is established through empirical Sutherland law. The thermal conductivity K (T) is obtained assuming that the molecular Prandtl number is 0.7 .

\section{Large eddy simulation}

Equations are closed from Large Eddy Simulation technique. It means simulate directly all large scale in the flow without need a turbulence model. At the same time, these large scales will be filtered larger in a local mesh size and its effect on the movement of small scales are modelled after with a sub-mesh model. For filtered equations and sub-mesh terms, see [8]. The sub-grid scale model used is the selective structure function model, which is an extrapolation to the physical space of the spectral model [9].

\subsection{Numerics}

The code used in this work was developed in Engineering Institute of UNAM and it has already been tested to simulate different turbulent flows $[8,9,10,11]$. This in-house code use a generalized coordinate system and is solved by an extension of the explicit scheme McCormack, second order in time and fourth order in space, developed by Gottlieb and Turkel. Adaptation to the generalized coordinates is performed by introducing a Jacobian matrix. It transforms a complex or curvilinear geometry in a Cartesian coordinate system mesh in a simple orthogonal geometry with uniform mesh. This scheme is a predictor-corrector scheme.

\subsection{Flow configuration, mesh and boundary conditions}

The geometry is similar to inverse diffusion flame burner. It only was considered like a mixing chamber and simulated as non-reactive flow. Central round jet is injected in a quiescent environment, Figure 2. At the same time four annular jets inject fluid into the chamber. Central jet remain at constant velocity $\left(U_{j}\right)$ and annular round jets were tested with different velocities $\left(U_{a}\right)$. Velocity ratios $\left(U_{a} / U_{j}\right)$ were $0.5,1.0$ and 2.0 times of main jet velocity (Table 1$)$.
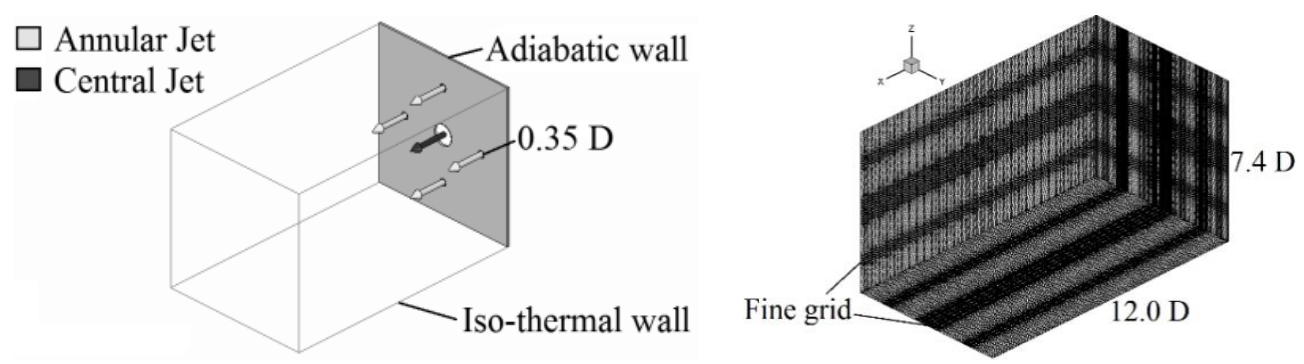

Fig. 2: Geometry and computational domain of mixing chamber. 
Table 1: Simulated cases.

\begin{tabular}{|c|c|}
\hline Case & Velocity ratios $\left(\boldsymbol{U}_{\boldsymbol{a}} / \boldsymbol{U}_{\boldsymbol{j}}\right)$ \\
\hline C1 & 0.5 \\
\hline C2 & 1.0 \\
\hline C3 & 2.0 \\
\hline
\end{tabular}

Reynolds number $=10800$, the Mach number $=0.3$ (subsonic flow) and the Prandtl number $=0.7$. Only a 12 diameters on axial direction of the chamber is simulated to save time calculation. Injection has a top-hat profile. Boundary conditions proposed by [12] are applied and used with fixed temperature and velocity at inlet, and prescribed pressure for outlet. Walls are considered as adiabatic walls.

\section{Code validation}

The validation code was performed using a baseline case (BC). BC represent single round turbulent jet. Numerical results were compared with the experimental data of [3]. After initial and transitional region, the flow develops selfpreserving behavior. Self-similarity region was analysed. The inverse of velocity profile along the axis present a linear behaviour.

$$
\frac{U_{0}}{U_{c}}=\frac{1}{B e}\left(\frac{x-x_{0}}{D}\right)
$$

$U_{0}$ is exit velocity, $U_{c}$ is centreline mean velocity and $\frac{x_{0}}{D} \approx 4$, where $x_{0}$ is the virtual origin. Slope value of decrease of velocity in self-similar region is presented in the Table 2. Although there is a change in slope it shows a good agreement with experimental data. Also, velocity and steamwise turbulence intensity profiles were compared, Figure 3.

Table 2: Centreline mean velocity parameter, $1 / B e$.

\begin{tabular}{|c|c|c|}
\hline Self-similar region & Hussein (1994) & Present work \\
\hline 1/Be & 0.1724 & 0.1743 \\
\hline
\end{tabular}
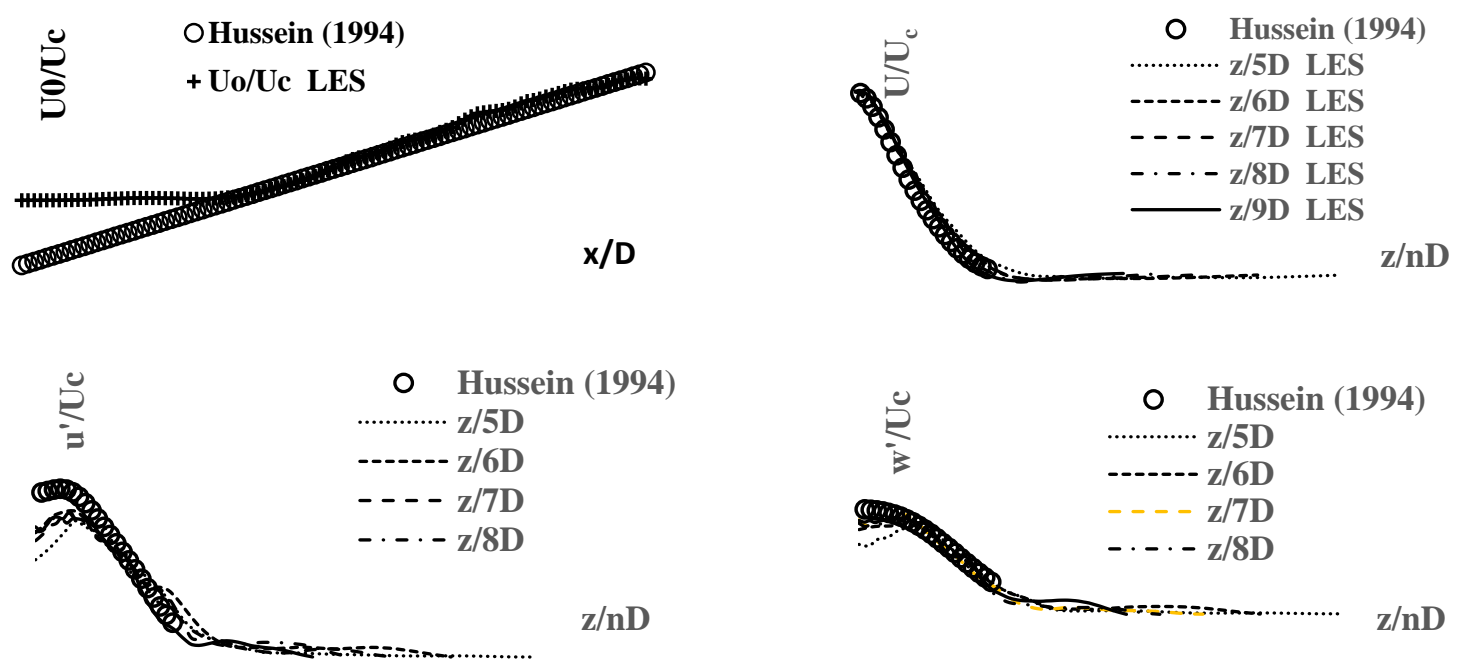

Fig. 3: Centreline inverse mean velocity profile (top-left), steamwise mean velocity profile (top-right), steamwise turbulence intensity profile (Bottom-left) and radial turbulence intensity (bottom-right). 


\section{Results}

\subsection{Vortex structures}

As a first analysis vortex structures visualization was performed using $Q$ criterion, Figure 5. For BC, when the jet leave the nozzle amplified perturbation are developed. Furthermore, the entrain fluid received and spread in radial direction downstream produce an increase on size until the initial momentum is dissipated by viscous effects [13]. In Figure 5, in $\mathrm{C} 1$ the peripheral jets cause a change in the vortex zone size within the first-half (axial length) of the central jet, this is due to turbulent interactions. Additionally, in the second-half, no modifications are observed. Turbulent region conserve similar behaviour as BC. In the following cases $(\mathrm{C} 2, \mathrm{C} 3)$ an increase in the velocity also produce an increase in turbulence intensity of peripheral jets. C3 present a particular feature, the increase in the intensity causes an apparent contraction that affects the development of the central flow.

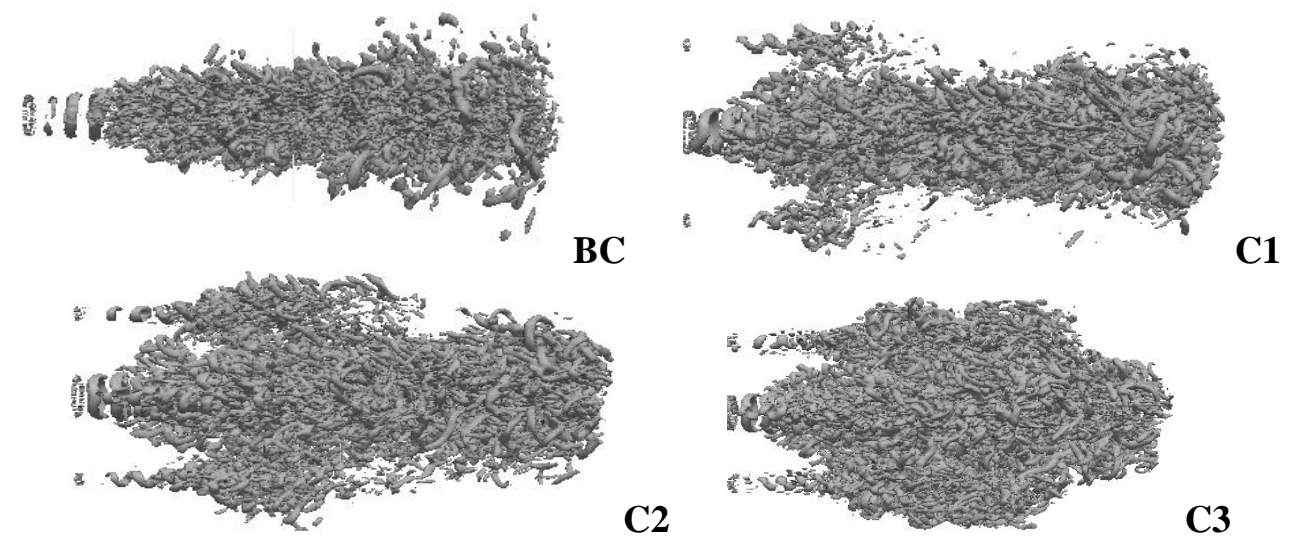

Fig. 5: Iso-surface of $Q$ criterion, $Q=30.0$.

An instantaneous iso-contour of concentration is presented in Figure 6. All cases present coherent structures at outside of the jet. However, cases with peripheral jets shows earlier transition to turbulence and mixing. Consequently, C3 has a shorter potential core, Figure 7. The difference between the main jet and the annular jet velocity seems to aid the development of turbulence and affects the mixing efficiency.

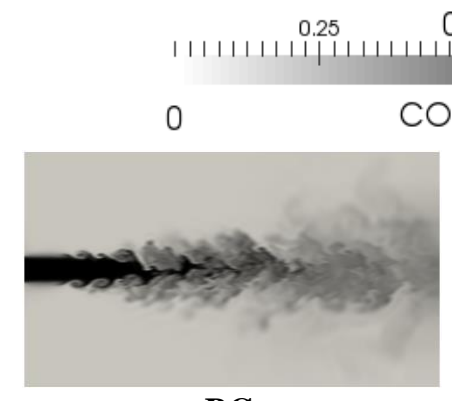

BC

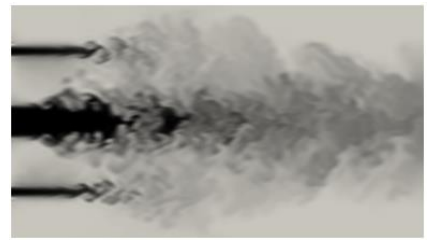

C2
$0.5 \quad 0.75$
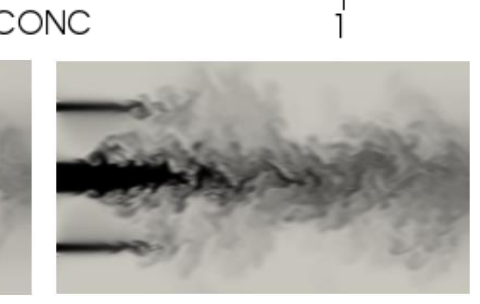

C1

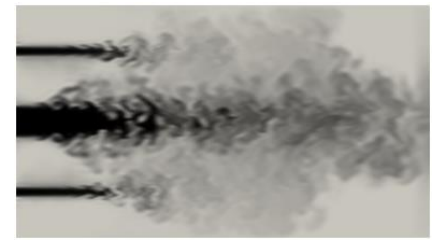

C3
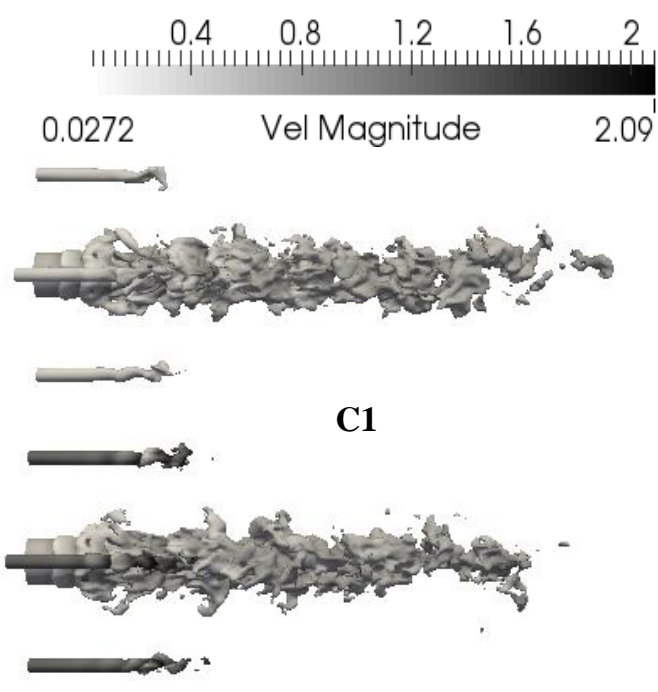

C3

Fig. 6: Snapshot of concentration in the middle section of axial length (left) and iso-surface of concentration $c=0.5$ with contour of the magnitude of velocity for $\mathrm{C} 1$ and $\mathrm{C} 3$ (right). 


\subsection{Anisotropy Invariant Map (AIM)}

To characterize the main jet turbulence state, Lumley-Newman representation was used $[14,15,16]$, it is based on the use of anisotropic invariant of the Reynolds stress tensor $\left(a_{i j}\right)$, and is obtained by deviatoric tensor $\left(b_{i j}\right)$

$$
a_{i j}=\frac{b_{i j}}{k_{e c}}=\frac{\overline{u_{\imath}^{\prime} u_{j}^{\prime}}}{k_{e c}}-\frac{2}{3} \delta_{i j}
$$

where $k_{e c}$ represents turbulent kinetic energy and $u^{\prime}$ are the rms-fluctuations of the velocity. This tensor is the basis of turbulence anisotropy analysis. With the second (I2) and third (I3) invariant defined as follow

$$
\begin{gathered}
I 2=a_{i j} a_{j i} \\
I 3=a_{i j} a_{j k} a_{k i}
\end{gathered}
$$

the Lumley triangle is established to determine the degree of anisotropy and its nature. For analysis, anisotropy invariant maps were obtained from three different regions along the axial length for each case, Figure 7.

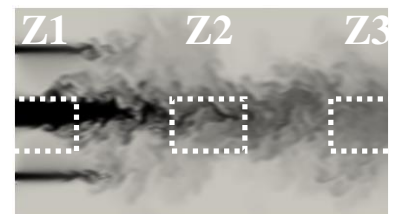

Fig. 7: Analyzed zones: Z1 - initial, Z2 - middle and Z3 - final.
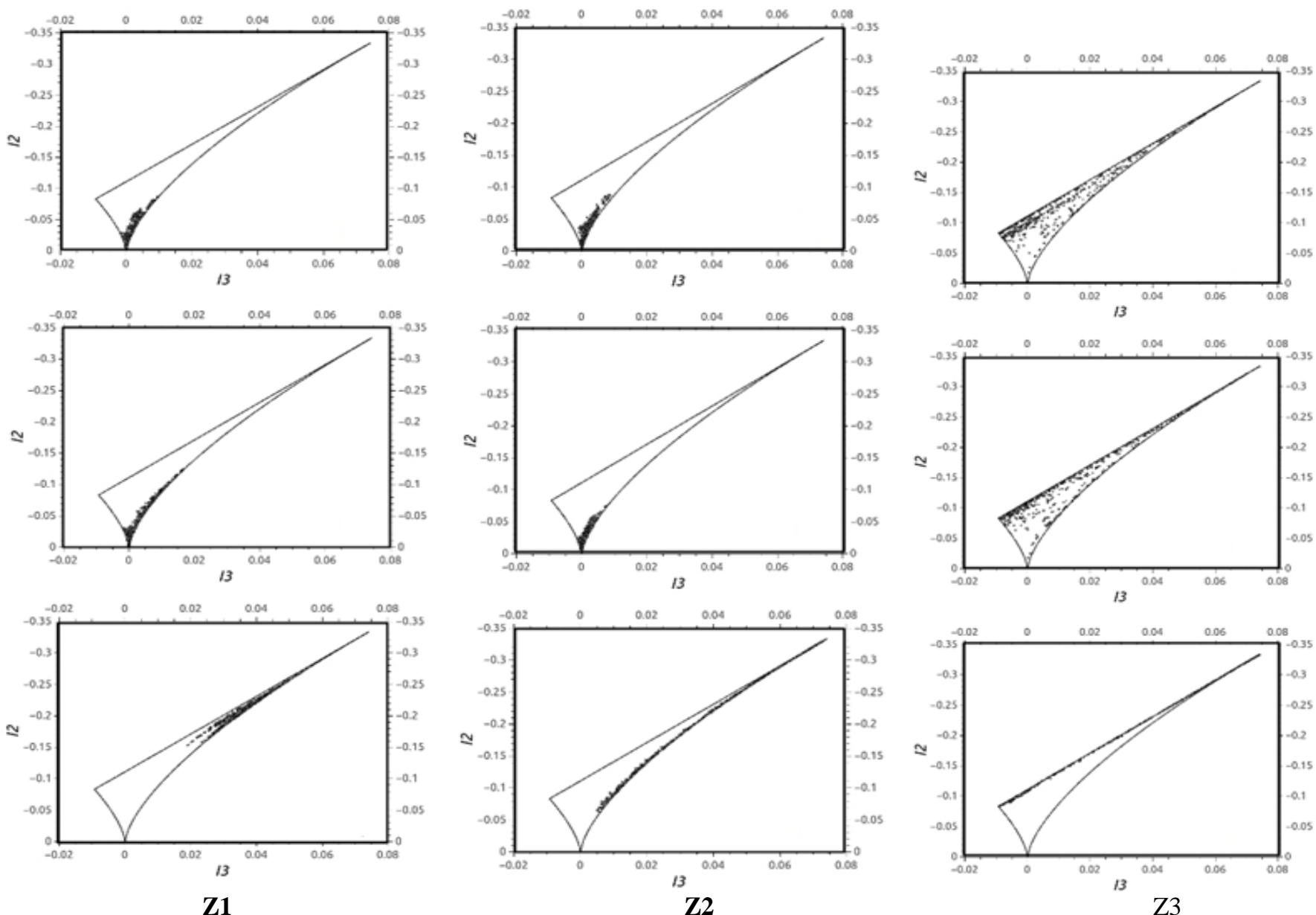

Fig. 8: Anisotropy invariant maps of analysed zones. 
Results of all analysed zones are presented in Fig. 8. In Z3, near of the outlet chamber: C2 is not significantly affected by the peripheral jets and behaves similar to BC. In BC turbulence has not a well-defined state. Points are scattered throughout the triangle area. However, the state tends to be axisymmetric of two components. In C3, the behaviour changes from two components to a single component. This implies that the peripheral jets influence the state of turbulence downstream of the chamber (the potential core is also affected).

In Z1y Z2, C2 is not influenced significantly by the peripheral jets. However, turbulence is more homogeneous and isotropic. This case tends to an axisymmetric behaviour of two components with a form of "cigarette". This behaviour is common in free shear layers and jets. In contrast, in C3, turbulence sate passes of two components to a single component. This means that peripheral jets generate significant decrease in the radial fluctuations. The annular jets act as walls over central jet.

\section{Conclusion}

Three strongly confined turbulent round jet with four annular jets have been studied by LES and anisotropy invariant map. The velocity ratios range from 0.5 to 2.0. In this work, the effect of annular jets over central jet, the degree of anisotropy and the nature of turbulence is analysed.

Velocity ratios greater or equal to 1 intensify turbulent region in the stream. This causes increased interaction between the central jet and annular jets. Analysis shows reduction of potential core in central jet when the peripheral jets have higher velocities than the central jet velocity. However, peripheral jets generate a significant decrease in radial fluctuations. It means that the annular jets act as walls and confine the central jet. This could adversely affect the mixing process.

\section{Acknowledgements}

The authors appreciate the financial support provided by National Autonomous University of Mexico, PAPIIT DGAPA IA103515. The authors gratefully acknowledge the support from Ricardo Del Rio Serrano, Jonathan Hernández García, Ana Karen Moreno Álvarez and Martha Luisa Preciado Méndez.

\section{References}

[1] M.T. Kandakure, V.C. Patkar and A.W. Patwardhan, "Characteristics of turbulent confined jets," Chemical engineering and processing: Process intensification., vol. 47, no. 8, pp. 1234-1245, 2008.

[2] P. Wang, J. Fröhlich, V. Michelassi, and W. Rodi, "Large-eddy simulation of variable-density turbulent axisymmetric jets," J. Heat and Fluid Flow., vol. 29, pp. 654-664, 2008.

[3] H.J. Hussein, S.P. Capp, and W.K. George, "Velocity measurements in a high-Reynolds-number, momentum conserving, axisymmetric, turbulent jet," J. Fluid Mech., vol. 258, pp.31-75, 1994.

[4] T.H. Weisgraber and D. Liepmann, "Turbulent structure during transition to self-similarity in a round jet," Exp. Fluids., vol. 24, pp. 210-224, 1998.

[5] D.R. Webster, P.J.W. Roberts, and L. Ra'ad, "Simultaneous DPTV or PLIF measurement of a turbulent jet," Exp. Fluids., vol. 30, pp. 65-72, 2001.

[6] G. Singh, T. Sundararajan, and K.A. Bhaskaran, "Mixing and entrainment characteristics of circular and noncircular confined jets," J. Fluids Eng., vol. 125, pp. 835-842, 2003.

[7] Kandakure et al. "Characteristics of turbulent confined jets," J. Chemical Engineering and Processing, vol. 47, pp. 1234-1245, 2008.

[8] M. Salinas-Vázquez et al. "Large Eddy Simulation of a jet flow with a suction/ejection system using immersed boundary conditions," Journal of Wind Engineering and Industrial Aerodynamics, vol. 98, pp. 618-627, 2010.

[9] M. Salinas-Vázquez and O. Métais, "Large-eddy simulation of the turbulent flow through a hearted square duct," $J$. Fluid Mech, vol. 453, pp. 201-238, 2002.

[10] M. Salinas-Vázquez et al. "Large Eddy Simulation of a flow through circular tube bundle," Applied Mathematical Modelling, vol. 35, pp. 4393-4406, 2011.

[11] M. Salinas-Vázquez et al. "A low-Mach number method for the numerical simulation of complex flows," Applied Mathematical Modelling, vol. 37, pp. 9132-9146, 2013. 
[12] T. Poinsot and S. Lele, "Boundary conditions for direct simulation of compressible viscous flows," J. Comput. Phys., vol. 101, pp. 104-129, 1992.

[13] C.G. Ball, H. Fellouah, and A. Pollard, "The flow field in turbulent round free jets," Progress in Aerospace Sciences, vol. 50, pp. 1-26, 2012.

[14] A. J. Simonsen and P.A. Krogstad, "Turbulent stress invariant analysis: clarification of existing terminology," in Proceedings of Australasian Fluid Mechanics Conference of the University of Sydney. Sydney, Australia, 2004, vol. 15, pp. 13-17.

[15] R. Escudié and A. Liné. "Analysis of turbulence anisotropy in a mixing tank," J. Chemical Engineering Science, vol. 61, pp. 2771-2779, 2006.

[16] Radenković Darko R. et al. "Anisotropy analysis of turbulent swirl flow," in FME Transactions, vol. 42, pp. 19-25, 2014. 